\title{
Criminologie
}

\section{La vidéosurveillance, une réponse efficace à la criminalité ?}

\section{Éric Heilmann}

Volume 36, numéro 1, printemps 2003

Police et prévention : évaluation et analyse d'impact

URI : https://id.erudit.org/iderudit/006554ar

DOI : https://doi.org/10.7202/006554ar

Aller au sommaire du numéro

Éditeur(s)

Les Presses de l'Université de Montréal

ISSN

0316-0041 (imprimé)

1492-1367 (numérique)

Découvrir la revue

Citer cet article

Heilmann, É. (2003). La vidéosurveillance, une réponse efficace à la

criminalité ? Criminologie, 36(1), 89-102. https://doi.org/10.7202/006554ar

\section{Résumé de l'article}

Cet article présente les principales observations formulées par les criminologues qui ont analysé l'impact de la vidéosurveillance sur la délinquance urbaine en Grande-Bretagne. Les stratégies d'évaluation utilisées par les chercheurs ont permis de montrer qu'il varie sensiblement selon le contexte d'implantation et d'exploitation des dispositifs techniques. Quatre conditions au moins doivent être réunies pour que la vidéosurveillance produise des effets : l'allocation de ressources suffisantes, la prise en compte de la configuration du tissu urbain, le soutien par d'autres mesures préventives et la définition de cibles pertinentes. 


\title{
La vidéosurveillance, une réponse efficace à la criminalité ?
}

\author{
Éric H eilmann \\ Maître de conférences \\ Université Louis Pasteur de Strasbourg \\ France \\ eric.heilmann@urfist-ulp.u-strasbg.fr
}

RÉSUmÉ - Cet article présente les principales observations formulées par les criminologues qui ont analysé l'impact de la vidéosurveillance sur la délinquance urbaine en Grande-Bretagne. Les stratégies d'évaluation utilisées par les chercheurs ont permis de montrer qu'il varie sensiblement selon le contexte d'implantation et d'exploitation des dispositifs techniques. Quatre conditions au moins doivent être réunies pour que la vidéosurveillance produise des effets: I'allocation de ressources suffisantes, la prise en compte de la configuration du tissu urbain, le soutien par d'autres mesures préventives et la définition de cibles pertinentes.

\begin{abstract}
The impact of cCTV systems on crime rates has been more extensively researched in Great Britain than anywhere else. We offer, in this paper, a review of the findings of this systematic effort in evaluation research. The main finding is that a number of necessary conditions must be satisfied for a significant prevention effect to occur: first, an appropriate mobilization of resources invested in the experiments; second, a conceptually adequate specification of relevant, feasible and measurable target outcomes; third, a package of auxiliary but necessary treatment components; fourth, a thorough understanding of the unique ecological attributes of the experimental and control environments. A recurrent pattern in cctv and crime research is that these technological solutions are in fact sensitive to the implementation and actual management contingencies that shape their utilization.
\end{abstract}

Après plusieurs années passées à étudier les usages policiers de la vidéosurveillance, Jason Ditton, directeur du Scottish Centre for Criminology, a fini par conclure que l'implantation de tels dispositifs techniques pouvait jouer un rôle actif dans la prévention et la gestion des désordres urbains. 
Et d'inviter in fine ses collègues chercheurs à s'intéresser plus sérieusement à la question de savoir non pas «si ça marche», mais «comment ça marche» (Ditton, 1999a). Les études menées sur ce thème depuis le début des années 1990 en $\mathrm{G}$ rande-B retagne permettent déjà d'apporter quelques éléments de réponse ${ }^{1}$.

Pourquoi s'intéresser à la Grande-Bretagne? De façon pragmatique, on peut avancer trois raisons pour justifier cette attention singulière. La première est qu'il s'agit du pays qui compte actuellement le plus d'équipements de vidéosurveillance au sein de l'U nion européenne. La seconde est que l'installation des systèmes s'est réalisée dans un même cadre politique de référence, c'est-à-dire à la suite du lancement d'une série d'appels à projets (CCTV Challenge Competition) financés par le gouvernement, le Home $O$ ffice en Angleterre et au pays de Galles et le Scottish 0 ffice en Écosse. L'opération a permis le développement tout azimut des dispositifs implantés dans les villes: 39 sont équipées en 1993, 79 en $1994 \ldots$ quatre ans plus tard, toutes les grandes villes de plus de 500000 habitants (sauf Leeds) et près de 400 villes de taille moyenne ont installé de tels équipements sur la voie publique (voir H orne, 1996; N orris et Armstrong, 1999). Enfin, pour l'observateur français, les travaux des criminologues britanniques présentent un intérêt supplémentaire dans la mesure où aucune recherche sur ce sujet n'a jamais été réalisée en France. En effet, de ce côté de la $M$ anche, les spécial istes des problèmes sécuritaires ont surtout alimenté le débat qui oppose de façon traditionnelle les défenseurs des libertés face à la menace technologique aux partisans d'un renforcement des moyens de protection des biens et des personnes. D eux points de vue irréductibles, qui reposent néanmoins sur une même croyance: I'efficacité des systèmes mis en œuvre qui, au demeurant, n'est jamais discutée. Q ue les coûts d'installation et de fonctionnement des systèmes de vidéosurveillance n'aient cessé de croître au fil du temps n'y change rien. De même les données publiées dans la presse, qui se fait régulièrement l'écho de déclarations grandiloquentes de tel ou tel maire ou chef de police municipale, ne font que refléter le déficit d'information en la matière.

En Grande-B retagne, des chercheurs professionnels ont conduit des études pour évaluer l'efficacité de la vidéosurveillance, certaines commanditées par le Home 0 ffice dans le métro londonien (Webb et Laycock,

1. Pour des études plus anciennes, voir en particulier Musheno et al. (1978) et Burrows (1979). 
1992), dans les parkings de villes de province (Tilley, 1993) et dans les centres-villes de N ewcastle, Birmingham et King's Lynn (Brown, 1995); d'autres par des institutions locales, comme à Brighton (Squires et M easor, 1996), à D oncaster (Skinns, 1998), à Airdrie et à Glasgow (Ditton et Short, 1995a; 1998a; 1999). Point commun de tous ces travaux : un recours systématique à des méthodologies éprouvées dans le champ des sciences criminelles qui donnent à leurs résultats une valeur scientifique peu contestable.

Si le gouvernement britannique, en soutenant massivement l'implantation de caméras de surveillance dans les espaces urbains, était convaincu au début des années 1990 qu'il détenait là une arme efficace pour lutter contre la criminalité, dix ans plus tard, ces études de terrain indiquent que I'enthousiasme originel mériterait d'être fortement tempéré. Tous les auteurs s'accordent en effet pour affirmer que la vidéosurveillance ne peut être efficace que si un certain nombre de conditions sont réunies.

\section{1. À propos des stratégies méthodologiques d'évaluation}

Force est de constater que Ditton et Short (1995b) ont formulé les critiques les plus sévères à l'égard des travaux menés jusque-là par des organisations locales (municipalité, service de sécurité, industriel, etc.) pour évaluer l'efficacité des équipements mis en œuvre. Plusieurs raisons sont avancées par les auteurs pour expliquer l'absence de crédibilité de ces «self-evaluations», comme ils les désignent:

- les chiffres communiqués pour apporter la preuve de l'efficacité des dispositifs sont invariablement exprimés en termes de pourcentage, ce qui ne permet pas de connaître la réalité d'un phénomène: plus le nombre de délits est faible, plus les fluctuations seront grandes, « une baisse de $25 \%$, soulignent-ils, traduit simplement que l'on est passé de quatre à trois délits» ;

- plusieurs types de délits sont pris en compte, mais agrégés dans un même ensemble statistique, de sorte qu'il est impossible d'évaluer l'impact de la vidéosurveillance selon la nature du délit;

- la période de temps qui sert de référence aux enquêtes est trop courte et ne permet pas de prendre en compte les fluctuations (avant et après l'installation des systèmes) des taux de criminalité dans une local ité et ses alentours, ou encore de mesurer le caractère durable d'une baisse éventuelle; 
- aucun espace de contrôle n'a été défini pour suivre l'évolution de la délinquance dans des zones voisines non équipées de caméras et pour établir, le cas échéant, l'existence d'un déplacement de la criminalité. Pour les criminologues qui s'intéressent aux protocoles d'évaluation des projets de prévention situationnelle, ces problèmes sont déjà connus?. Reste à savoir de quelle façon précise ils ont été pris en compte par les chercheurs qui étudient l'impact de la vidéosurveillance dans les espaces urbains.

\section{La prévention de quels délits?}

Dans l'enquête qu'ils mènent à Airdrie, petite cité de 35000 habitants située à l'est de G lasgow, D itton et Short (1995a) analysent les données recueillies par la police 24 mois avant et après l'installation des caméras, dans six zones de référence: les rues du centre-ville placées sous surveillance vidéo, les autres quartiers du centre-ville non équipés de caméras, puis des zones de plus en plus larges (à l'exclusion des deux précédentes) qui correspondent à des circonscriptions administratives de police (division, district, etc.). Un découpage qui s'apparente à un jeu de poupées russes. Q ui plus est, sept catégories différentes d'infractions sont prises en compte ${ }^{3}$.

Ce protocole permet aux auteurs d'effectuer une analyse extrêmement fine de l'impact de la vidéosurveillance. Si le nombre de crimes et délits (toutes catégories confondues) a diminué de $21 \%$ dans les espaces couverts par les caméras, ils relèvent en effet que ce chiffre varie sensiblement selon le type d'infractions:

- la catégorie qui inclut les cambriolages, les vols de (ou dans les) véhicules et les vols à l'étal age ( «crimes of dishonesty »), a connu le taux de réduction le plus élevé (- $48 \%$ ), ce qui représente près de 1230 délits en moins par rapport aux 24 mois qui ont précédé l'installation du système;

- le nombre d'actes de vandalisme a chuté de $19 \%$ au cours de la période de référence (42 délits en moins);

- les deux catégories qui incluent les infractions les plus graves, comme les homicides, les crimes sexuels, les agressions violentes ou les

2. Voir notamment Rosenbaum, 1988 ; Ekblom et Pease, 1995 ; Crawford, 1998.

3. Toutes les données brutes recueillies auprès des services de police ont été corrigées pour tenir compte des variations saisonnières. 
attaques à main armée ("crimes of violence and crimes of indecency »), n'ont pas connu d'évolution notable, leur nombre étant demeuré stable (une centaine avant et après I'installation des caméras);

- le nombre de délits relatifs à l'usage de drogues a augmenté de $106 \%$ au cours de la période de référence (180 délits supplémentaires);

- le taux des infractions relatives à l'usage de véhicules (conduite en état d'ivresse, excès de vitesse, etc.) a augmenté de $126 \%$ (128 délits supplémentaires);

- la catégo rie qui regroupe les atteintes à la paix publique (bagarre, état d'ivresse, etc.) a connu le taux de croissance le plus élevé (+ $133 \%$ ), ce qui représente 194 délits supplémentaires.

C'est là un des principaux enseignements de cette enquête: la vidéosurveillance a des effets distincts selon la nature des délits. Et toutes les études menées par ailleurs, en suivant une méthodologie identique, ne feront que confirmer cette observation.

Concernant les trois dernières catégories d'infractions, Ditton et Short soulignent encore que la forte augmentation de leur nombre ne signifie pas nécessairement que la vidéosurveillance n'a pas eu d'effet dans ce domaine. Comme d'autres auteurs (Squires et M easor, 1996), ils avancent I'hypothèse que la présence des caméras a pu accroître le nombre d'incidents détectés par les opérateurs et augmenter en conséquence les statistiques criminelles. C'est dire que cet effet de reportabilité est un biais qui peut conduire les observateurs à sous-estimer l'efficacité imputée à la vidéosurveillance.

Par ailleurs, Ditton et Short considèrent qu'il n'y a aucune «évidence statistique» permettant d'établir l'existence d'un déplacement de criminalité ${ }^{4}$. Cela dit, ils n'écartent pas cette hypothèse mais suggèrent plutôt d'utiliser d'autres méthodes d'investigation - comme des entretiens avec des délinquants pour connaître leurs motivations et leurs modes d'action - afin de saisir la réalité du phénomène $e^{5}$. Ils réalisent ce travail quelques mois plus tard en conduisant une série d'entretiens avec des délinquants qui ont comparu devant le tribunal de grande instance ("Sheriff Court») d'Airdrie (1998b). O n notera simplement ici, s'agissant des atteintes à la propriété, que la majorité des délinquants affir-

4. En revanche, un phénomène de déplacement géographique de la criminalité est constaté à Birmingham par Brown (1995) et à Doncaster par Skinns (1998) pour les délits classés dans la première catégorie (cambriolage, vol à l'étalage, etc.).

5. Dans le même sens, voir Gabor (1990:60). 
ment que la vidéosurveillance a eu un impact sur leur comportement dans la mesure où ils ont agi avec plus de prudence... mais sans pour autant renoncer à commettre des délits. Certains prétendent que la présence des caméras n'a rien changé à leur manière d'agir. Q uelques personnes reconnaissent s'être déplacées ailleurs, la ville la plus souvent mentionnée étant celle de Glasgow (située à 15 milles d'Airdrie). Ditton et Short ajoutent au passage que les délinquants qui étaient soupçonnés de voyager dans l'autre sens, c'est-à-dire de Glasgow à Airdrie, pour commettre des vols, semblent désormais voyager encore plus loin...

\section{Pour une méthode «réaliste» d'évaluation}

Auteur du G uide d'utilisation de la vidéosurveillance diffusé en 1994 par le H ome $O$ ffice auprès des candidats aux CCTV Challenge Competitions, Tilley a inspiré bon nombre de chercheurs sur le plan méthodologique. L'enquête qu'il consacre à la vidéosurveillance dans les parkings de six villes de province est la première du genre en Grande-Bretagne au regard de l'ampleur géographique et temporelle de l'éval uation (Tilley, 1993). C'est à cette occasion qu'il explicite les bases de sa «realistic evaluation method » qui s'articule autour de l'examen de trois éléments: les «mécanismes» («mechanisms») par lesquels la vidéosurveillance peut avoir un effet sur l'évolution de la criminalité, le «contexte» («context») d'utilisation de la technologie dans un lieu donné et les « résultats tendances» ( «outcome pattern») qui sont produits par ces mécanismes dans des conditions particulières (voir aussi Pawson et Tilley, 1997).

Parmi ces mécanismes, qui sont autant d'hypothèses concernant la façon dont des mesures de prévention peuvent produire des effets, Tilley relève en particulier le redéploiement du personnel de sécurité dans les espaces visés par les caméras et à proximité, la publicité accordée aux arrestations rendues possibles par les enregistrements vidéo, ${ }^{6}$ la présence de panneaux informant le public de l'existence de caméras, autant d'éléments qui peuvent dissuader un délinquant potentiel de commettre un délit, mais aussi rassurer les usagers et les encourager à fréquenter les stationnements placés sous surveillance. À l'inverse, souligne l'auteur, on

6. À Newcastle, Brown (1995) fournit un exemple concret de l'impact dissuasif de la publicité : parmi tous les délits étudiés, seuls les cambriolages ont connu une baisse sensible et durable, mais la période durant laquelle elle a été la plus forte est celle qui va de décembre 1992 à mars 1993, c'est-à-dire entre le moment où l'installation du système est annoncée dans les médias et celui où sa mise en œuvre est effective... 
peut très bien imaginer que la présence de la vidéosurveillance amène le personnel de sécurité, trop confiant dans la capacité de l'appareillage technique, à relâcher son attention ou encore conduise les délinquants à se déplacer et à agir dans des espaces non équipés. Plusieurs mécanismes peuvent se combiner.

Les éléments contextuels à prendre en compte sont également variés: les conditions d'éclairage et d'utilisation du stationnement (fréquentation, horaire, durée de l'immobilisation), le nombre et la performance des caméras, le comportement des usagers relatif à la sécurité de leur véhicule, la capacité de réaction du personnel de sécurité ou de la police face à la survenance d'un incident, etc. Ces derniers éléments peuvent évoluer et être adaptés au fil du temps pour permettre aux responsables des lieux placés sous surveillance d'atteindre, avec plus ou moins de succès, les objectifs qu'ils se sont fixés.

Q uant à l'analyse des résultats, elle repose sur l'examen des données quantitatives fournies par les services de police, mais aussi des données qualitatives recueillies au cours d'entretiens avec les responsables d'exploitation, les agents de sécurité ou les usagers (voir aussi Beck et Willis, 1995).

Cette stratégie d'évaluation permet à Tilley de souligner que les mécanismes associés à l'implantation de la vidéosurveillance sont multiples et ne produisent pas les mêmes effets selon le contexte d'utilisation du système. Les «résultats tendances» présentés dans son enquête témoignent bien de cette diversité: à Wolverhampton, les vols de voitures ont peu diminué dans le stationnement équipé de caméras et légèrement augmenté dans les autres stationnements de la ville; à Coventry, ils ont sensiblement diminué dans les cinq stationnements équipés, de même dans tous les autres stationnements de la ville; à B radford, ils ont fortement diminué dans le stationnement équipé, mais légèrement augmenté dans les deux stationnements voisins; à Hartlepool, ils ont nettement diminué dans les parkings équipés et augmenté par ailleurs, puis l'avantage des stationnements disposant de la vidéosurveillance a décliné avec le temps (environ dix-huit mois après I'installation des caméras).

\section{La vidéosurveillance, « comment ça marche? 》}

Q ue l'accent soit mis sur la prévention (baisse du volume de la délinquance) ou sur la répression (augmentation du taux d'élucidation), la vidéosurveillance n'est efficace que si certaines conditions sont réunies. 
On peut en identifier au moins quatre, comme en témoignent les exemples suivants.

\section{Allouer des ressources suffisantes et performantes}

Parmi les trois villes étudiées par Brown (1995), N ewcastle, Birmingham et King's Lynn, c'est dans cette dernière que la vidéosurveillance a eu l'effet dissuasif le plus marqué sur l'évolution de la délinquance. $M$ ais est-ce vraiment surprenant? Cette petite cité médiévale et marchande, qui compte près de 30000 habitants, dispose d'un système équipé de 60 caméras perfectionnées (soit une caméra pour 500 habitants), certaines disposant même de dispositifs infrarouges pour faciliter la vision de nuit. Les images sont transmises via un réseau de fibres optiques à un $P C$ vidéo équipé de 23 moniteurs. À Birmingham, qui compte plus de 900000 habitants (2,5 millions dans l'agglomération), seules 14 caméras sont implantées dans le centre-ville. Et l'auteur souligne de façon laconique que plusieurs ont été installées en dépit du bon sens: le champ de vision est obstrué, ici par du mobilier urbain, là par des arbres au feuillage abondant jusqu'à l'automne...

A Lewisham, Tilley (1993) relève qu'aucune arrestation n'a suivi la mise en œuvre de la vidéosurveillance dans un stationnement en raison de la pauvre qualité des images fournies par le système - sur les quatre caméras installées, une est un leurre, les trois autres ne disposent pas de zoom, de sorte qu'il est impossible d'identifier par ce canal les numéros d'une plaque d'immatriculation - et du nombre limité d'agents de sécurité disponibles pour intervenir après la constatation d'un incident.

Dans le même sens, le nombre et la qualification des opérateurs affectés à la surveillance des écrans ne sont pas étrangers aux performances d'un dispositif vidéo ${ }^{7}$. Une caméra traite 24 images par seconde, c'est-àdire plus de 2 millions d'images en 24 heures. Dans une ville qui compte dix caméras, par exemple, le système capte plusieurs dizaines de millions d'images au cours d'une journée! Ainsi, parmi les raisons invoquées pour expliquer pourquoi la vidéosurveillance semble être un succès à Airdrie et non à Glasgow, Ditton (1999a) souligne que deux agents sont présents en permanence pour assurer ce travail fastidieux dans les deux villes, mais

7. Sur le travail au quotidien des opérateurs, voir Norris et Armstrong (1999:91-201) qui ont passé près de 600 heures (!) dans des PC vidéo de trois métropoles différentes pour réaliser leur enquête. 
le dispositif compte 32 caméras dans la capitale écossaise et 12 caméras dans la petite cité voisine. Du reste, si le nombre d'incidents portés à la connaissance des opérateurs a nettement augmenté à Glasgow durant les douze mois qui ont suivi l'installation du système, leur intervention a entraîné un nombre limité d'arrestations: 290 ont été comptabilisées au cours de cette période, soit une arrestation par caméra tous les 40 jours...

\section{Prendre la mesure de la complexité de l'espace urbain}

La vidéosurveillance n'a pas un impact déterminant sur le volume de la délinquance dans les espaces complexes et étendus. C'est l'un des enseignements que l'on peut tirer de l'enquête conduite par Webb et Laycock (1992) dans le métro londonien. R appelons ici que la société qui gère le métro londonien a engagé, au milieu des années 1980, une série d'actions visant à renforcer la sécurité des usagers et des conducteurs de rame: installation de caméras dans des stations situées sur des lignes dites sensibles, implantation d'alarmes sur les quais, rénovation du réseau de communication assurant les liaisons radio entre les policiers et mise en œuvre d'un nouveau système de tickets « underground ticketing system » pour accéder aux stations, dont les plus importantes sont équipées de «barrières automatiques».

L'une des stations étudiées, 0 xford Circus, est l'une des plus fréquentées par les usagers: 250000 personnes y transitent quotidiennement. Sa configuration est particulièrement complexe puisqu'elle compte huit entrées/ sorties, six quais, quatorze tapis roulants et de multiples couloirs. Plusieurs mesures ont été prises dans cette station en avril 1988 : installation de 30 caméras reliées à un PC vidéo, implantation de 34 alarmes et création de quatre kiosques d'information pour recevoir les usagers. Les statistiques criminelles montrent néanmoins que le dispositif n'a pas permis de réduire la délinquance à 0 xford Circus: le nombre de vols à l'arraché est demeuré stable durant la période de référence (27 en 1985, 32 en 1988, 29 en 1989, 29 en 1990), celui des vols à la tire a légèrement augmenté au fil des ans (340, 397, 451 et 384 les mêmes années) et celui des agressions a connu une évolution en dents de scie $(13,35$, 32 et 16 les mêmes années).

Pour Webb et Laycock, ce résultat, plutôt décevant au regard des investissements consentis, s'explique en grande partie par la nature des délits constatés et la complexité de l'environnement à surveiller : la majorité des vols sont commis par des pickpockets dont l'action (rapide et 
discrète) peut aisément échapper à la vigilance d'un opérateur qui doit manipuler une trentaine de caméras à la fois. Et quand bien même il serait repéré à l'écran, l'auteur d'un vol pourrait facilement prendre la fuite dans les méandres de la station.

À l'inverse, sur la ligne N orthern qui a fait l'objet de la même attention (installation de nouvelles caméras, d'alarmes sur les quais et de kiosques d'information dans les six stations situées sur la ligne), les statistiques criminelles indiquent que le nombre de vols a diminué de moitié durant la même période de référence. Les auteurs rel èvent également que la baisse constatée après l'installation du dispositif n'a pas été suivie d'une augmentation dans les stations avoisinantes. Pour expliquer ce résultat, Webb et Laycock soulignent qu'un redéploiement des forces de sécurité a été organisé dans les stations situées au sud de la Tamise (N orthern, Victoria et Bakerloo) et que la configuration des stations de la ligne Northern n'a rien de comparable avec celle d'O xford Circus: chacune compte simplement deux quais, un tapis roulant, une entrée et une sortie. C'est dire que le risque d'être arrêté semble être assez grand pour dissuader un délinquant potentiel de passer à l'acte dans ce périmètre.

Les mêmes observations sont formulées par Brown (1995) et Ditton (1999a) lorsqu'ils comparent les résultats obtenus dans des villes de taille modeste (K ing's Lynn et Airdrie) et des métropoles (N ewcastle, Birmingham et Glasgow) où des caméras sont implantées pour couvrir des zones géographiques plus ou moins vastes et disparates.

\section{Combiner diverses mesures de prévention}

Tous les auteurs s'accordent pour affirmer que l'impact des dispositifs est plus significatif lorsque leur implantation est associée à d'autres mesures de prévention. Ainsi, à Brighton, Squires et M easor (1996) soulignent que le nombre d'agressions, visant en particulier des étudiants étrangers et des touristes, a fortement diminué durant la période estivale qui a suivi l'installation de 14 caméras dans le centre-ville. Si l'utilisation de la vidéosurveillance n'est pas étrangère à cette évolution, ils relèvent toutefois que la présence policière avait été renforcée sur le terrain durant cette période et qu'une campagne d'information ciblée avait été menée auprès des populations concernées.

8. Dans un contexte différent, K nutsson (1997) a montré que pour lutter avec succès contre le trafic de stupéfiants dans un parc de la ville de Stokholm, les autorités ont appliqué plusieurs mesures : élagage des haies, surveillance cachée, patrouilles ostensibles, etc. 
À Birmingham, Brown (1995) affirme que l'aménagement de rues piétonnes- qui a modifié les «conditions d'accès» des véhicules aux délinquants- a influé sur l'évolution à la baisse des vols de véhicules. À Doncaster, où près de 60 caméras ont été implantées dans les rues commerçantes et les principales artères du centre-ville, Skinns (1998) souligne que la forte baisse des vols de (et dans les) véhicules est liée à la politique adoptée par la municipalité, qui a restreint les espaces consacrés au stationnement automobile, et au redéploiement concomitant des forces de police au cœur de la cité.

À Coventry, Tilley (1993) relève que les vols de véhicules ont sensiblement diminué, et de façon continue, dans les cinq stationnements de la ville équipés de caméras (191 en 1987, 54 en 1992). II souligne toutefois que la municipalité a mis en œuvre d'autres mesures de prévention dans ces lieux durant la période de référence: coupe régulière du feuillage pour améliorer la surveillance, réfection des peintures et des éclairages, installation de panneaux pour informer les usagers de la présence de caméras, érection de hautes clôtures pour restreindre et canaliser l'accès des piétons. U ne alchimie singulière qui ne facilite pas la tâche des chercheurs-évaluateurs: Tilley conclut logiquement qu'il est impossible de savoir quelle mesure précise a pu avoir un effet déterminant dans chacun des stationnements étudiés.

\section{Définir des cibles et des objectifs pertinents}

La présence de caméras peut-elle exercer une influence sur le comportement d'une poignée de jeunes gens ivres qui veulent en découdre pour défendre leur «honneur» à la sortie d'un pub? O n peut sérieusement en douter. Comme le souligne justement Brown (1995), la vidéosurveillance n'a pas un effet dissuasif sur des individus dont le comportement est de nature impulsive. Toutes les enquêtes conduites dans des centres-villes indiquent d'ailleurs que les statistiques criminelles ont toujours évolué à la hausse après l'installation de caméras dans la catégorie qui regroupe les atteintes à la paix publique. $D$ ans un autre registre, Webb et Laycock (1992) ont montré que les auteurs de vols à la tire ne se sentaient guère menacés dans le métro londonien par la présence de caméras.

Bien que les criminologues britanniques ne se soient pas montrés très prolixes sur ce sujet, il faut bien admettre que la vidéosurveillance n'est pas une «machine à tout faire». Et si les marchands de biens de sécurité ont tout intérêt à entretenir cette idée pour faciliter la mobilisation des 
bailleurs de fonds potentiels autour de projets d'équipement, cette confusion des genres a des effets plutôt désastreux une fois les caméras en place: les opérateurs se retrouvent finalement seuls, contraints de discriminer jour après jour des individus et des évènements pour définir des cibles prioritaires et faire le choix d'une pondération entre des intérêts contradictoires. D'autant que, dans la pratique, s'ajoutent encore aux objectifs policiers ceux, éminemment politiques, des autorités locales qui ont financé les équipements pour restaurer l'image de leur cité, stimuler l'économie et apporter une réponse au sentiment d'insécurité des électeurs (voir Fyfe et Bannister, 1998 ; Coleman et Sim, 2000).

$0 r$, rien n'indique, dans les rapports étudiés ici, que des objectifs précis et réalistes ont été définis au préalable par les acteurs concernés. Sauf dans un cas, à Airdrie, comme le montre Ditton (1999a) en décrivant le processus qui a conduit à l'installation du système. $D$ ans la cité écossaise, elle est due à l'initiative du chef de la police locale en réponse à un problème posé dans un quartier par des jeunes qui se défendent d'être les auteurs d'actes de vandalisme et de vols à l'étalage. Le projet d'y installer des caméras est avancé puis étoffé par le chef de la police qui élabore un projet plus ambitieux et le soumet à l'ensemble de la population au cours de plusieurs réunions publiques. Parallèlement, un groupe de travail est constitué au sein du commissariat pour analyser les statistiques criminelles et définir les espaces devant faire en priorité l'objet d'une surveillance vidéo; une campagne d'information est ensuite menée en direction des commerçants en vue d'obtenir leur aide pour financer I'opération. Dans les jours qui ont suivi la mise en œuvre de la vidéosurveillance, Ditton relève que des centaines de personnes ont fait la queue durant plusieurs heures à l'entrée du PC vidéo pour jeter un œil à l'intérieur. C'est dire que les habitants d'Airdie sont «fiers de leurs caméras», comme le souligne l'auteur, mais aussi que les objectifs poursuivis sont clairs, limités et réalistes. R ien de tel à Glasgow, où la vidéosurveillance est présentée d'abord comme un moyen de restaurer l'image de cette vaste métropole dont l'activité industrielle est en déclin, objectif ambitieux mais à bien des égards irréaliste. Peu d'habitants résident dans le centre-ville et la majorité ignore la présence des caméras: selon une enquête réal isée à l'époque, seules $33 \%$ des personnes interrogées ont conscience de la présence des caméras trois mois après leur installation - $41 \%$ quinze mois après - alors même que les médias ont largement couvert l'événement (D itton, 1999b). 


\section{Conclusion}

Le temps de l'enchantement technologique est-il passé? En regard des attentes excessives et des investissements considérables engagés pour financer les équipements, les succès modestes de la vidéosurveillance pourraient être jugés décevants. Pourtant, il faut bien se rendre à l'évidence: il ne suffit pas de greffer des caméras dans l'espace urbain pour prévenir la délinquance. Un dispositif technique ne peut pas servir durablement à atteindre une multiplicité d'objectifs en même temps, avec la même intensité et les mêmes opérateurs aux commandes. Pour obtenir des résultats, une intervention même limitée doit s'inscrire dans un ensemble cohérent de mesures et/ ou d'actions sur le terrain. Une stratégie de ce type a été suivie à Airdrie après consultation et avec l'appui de la population. Et c'est là sans doute l'un des principaux enseignements de ces enquêtes: I'efficacité de la vidéosurveillance dépend autant (sinon plus) de la capacité des promoteurs des systèmes à mobiliser la population autour de leur projet que des moyens techniques et humains mis en œuvre. Ditton laisse entendre en effet que les habitants, les opérateurs et leurs employeurs doivent construire ensemble une destination sociale à l'objet technique. En l'absence d'une telle négociation, les usages policiers de la vidéosurveillance risquent fort d'être incompris et rejetés.

\section{Références}

Beck, A., \& Willis, A. (1995). Crime and security. M anaging the risk to safe shopping. Leicester : Perpetuity Press.

Brown, B. (1995). Closed circuit television in town centres: three case studies. Crime Prevention Unit Series Papers, $n^{\circ} 68$. Londres: Home 0 ffice.

Burrows, J.N. (1979). The impact of CCTV on crime in the London Underground. In P. M ayhew, R.V. Clarke, J.N. Burrows, J.M. Hough \& W.C. W inchester (eds), C rime in Public View. H ome 0 ffice R esearch Study, $n^{\circ} 49$. Londres: H ome 0 ffice.

Coleman, R., \& Sim, J. (2000). «You'll never walk alone»: CCTV surveillance, order and neo-liberal rule in Liverpool city centre. British Journal of Sociology, 51 (4), 623-639.

Crawford, A. (1998). Crime, prevention \& community safety. Londres: A. W. Longman.

Ditton, J. (1999a). Why does CCTV seem to work in Airdrie but not in Glasgow? CCT V Today, 6 (5), 10-13.

Ditton, J. (1999b). What do Glaswegians think of their city centre CCTV cameras? CCT V Today, 6 (6), 15-19.

Ditton, J., \& Short, E. (1995a). D oes CCT V prevent crime? A n evaluation of the use of CCT V surveillance cameras in Airdrie town center. Crime and Criminal 
Justice Research Findings, $n^{\circ} 8$. Édimbourg: The Scottish 0 ffice Central Research Unit.

Ditton, J., \& Short, E. (1995b). D oes CCTV affect crime? CCT V Today, 2 (2), 10-12.

Ditton, J., \& Short, E. (1998a). Evaluating Scotland's first town centre CCTV scheme. In C. N orris, J. M organ \& G. Armstrong (eds), Surveillance, CCTV and social control (155-174). Aldershot: Ashgate Publishing.

Ditton, J., \& Short, E. (1998b). Seen and now heard. Talking to the targets of open street CCTV. British Journal of Criminology, 38 (3), 404-428.

Ditton, J., \& Short, E. (1999). T he effect of closed circuit tel evision cameras on recorded crime rates and public concern about crime in $\mathrm{G}$ lasgow. C rime and $\mathrm{Criminal}$ Justice Research Findings, $n^{\circ} 30$. Édimbourg: The Scottish Office Central Research Unit.

Ekblom, P., \& Pease, K. (1995). Evaluating crime prevention. In M. Tonry \& D. Farrington (eds), Building a safer society. C rime and justice, vol. 19. Chicago: University of Chicago Press.

Fyfe, N.R., \& Bannister, J. (1998). The eyes upon the street, CCTV and the city. In N.R. Fyfe (ed.), I mages of the street. Planning, identity and control in public space (254-267). Londres: Routledge.

Gabor, T. (1990). Crime displacement and situational prevention: toward the development of some principles. Canadian Journal of Criminology, 32 (1), 41-73.

H ome $O$ ffice (1994). CCT V : looking out for you. Londres: H ome $O$ ffice.

Horne, C. J. (1996). The case for: CCTV should be introduced. International Journal of Risk, Security and Crime Prevention, 1 (4), 317-331.

K nutsson, J. (1997). Restoring public order in a city park. Crime Prevention Studies, 7, 13-52.

M usheno, M .C., Levine, J., \& Palumbo, D. (1978). Television surveillance and crime prevention : evaluating an attempt to create defensible space in public housing. Social Science Q uarterly, 58 (4), 647-656.

N orris, C., \& Armstrong, G. (1999). T he maximum surveillance society. The rise of CCT V. Oxford: Berg.

Pawson, R ., \& Tilley, N. (1997). Realistic evaluation. Londres: Sage.

Rosenbaum, D.P. (1988). Community crime prevention: a review and synthesis of the literature. Justice Q uarterly, 5 (3), 323-393.

Skinns, D. (1998). Crime reduction, diffusion and displacement: evaluating the effectiveness of CCTV in D oncaster. In C. N orris, J. M organ \& G. Armstrong (eds), Surveillance, CCT V and social control (175-188). Aldershot: Ashgate Publishing.

Squires, P., \& M easor, L. (1996). CCT V surveillance and crime prevention in Brighton. Health and Social Policy Research Centre, Brighton: University of Brighton.

Tilley, N. (1993). U nderstanding car parks, crime and CCT V : evaluation lessons from safer cities. Crime Prevention Unit Series Papers, $n^{\circ}$ 42. Londres: Home 0 ffice.

Webb, B., \& Laycock, G. (1992). Reducing crime on the L ondon underground. Crime Prevention U nit Paper $n^{\circ}$ 30. Londres: Home 0 ffice. 